\title{
The giant titin: how to evaluate its role in cardiomyopathies
}

\author{
Amar Azad $^{1,2} \cdot$ Giulia Poloni $^{1} \cdot$ Naeramit Sontayananon ${ }^{1} \cdot$ He Jiang ${ }^{1} \cdot$ Katja Gehmlich $^{1,3}$ (])
}

Received: 17 April 2019 / Accepted: 28 May 2019 / Published online: 30 May 2019

(c) The Author(s) 2019

\begin{abstract}
Titin, the largest protein known, has attracted a lot of interest in the cardiovascular field in recent years, since the discovery that truncating variants in titin are commonly found in patients with dilated cardiomyopathy. This review will discuss the contribution of variants in titin to inherited cardiac conditions (cardiomyopathies) and how model systems, such as animals and cellular systems, can help to provide insights into underlying disease mechanisms. It will also give an outlook onto exciting technological developments, such as in the field of CRISPR, which may facilitate future research on titin variants and their contributions to cardiomyopathies.
\end{abstract}

Keywords Titin · Animal models · Induced pluripotent stem cell derived cardiomyocytes · CRISPR/Cas9 - Genomeengineering $\cdot$ Cardiomyopathy

$\begin{array}{ll}\text { Abbreviations } \\ \text { CRISPR } & \begin{array}{l}\text { Clustered regularly interspaced short palin- } \\ \text { dromic repeat }\end{array} \\ \text { CRISPRa } & \text { CRISPR activation system } \\ \text { CRISPRi } & \text { CRISPR interference } \\ \text { DCM } & \text { Dilated cardiomyopathy } \\ \text { HCM } & \text { Hypertrophic cardiomyopathy } \\ \text { iPSC } & \text { Induced pluripotent stem cells } \\ \text { iPSC-CM } & \begin{array}{l}\text { Induced pluripotent stem cell derived } \\ \text { cardiomyocyte(s) }\end{array} \\ \text { TTNtv } & \text { Titin truncating variant(s) } \\ \text { PSI } & \begin{array}{l}\text { Percentage spliced in } \\ \text { RNAseq }\end{array} \\ \text { RNA sequencing }\end{array}$

Amar Azad and Giulia Poloni have contributed equally.

Katja Gehmlich

k.gehmlich@bham.ac.uk

1 Division of Cardiovascular Medicine, Radcliffe Department of Medicine and British Heart Foundation Centre of Research Excellence, University of Oxford, Oxford OX3 9DU, UK

2 Swansea University Medical School, Swansea SA2 8PP, UK

3 Institute of Cardiovascular Sciences, University of Birmingham, Birmingham B15 2TT, UK

\section{Introduction}

Titin, historically also known as connectin (Maruyama 1976), is the largest protein known: with 3.4-3.9 megadalton it is 100 times larger than an "ordinary protein", e.g. the house-keeping enzyme Glyceraldehyde 3-phosphate dehydrogenase, GAPDH. It is specifically expressed in striated muscle and has important structural and signalling functions for the contractile units, sarcomeres. Each titin molecule spans half a sarcomere from the Z-disc to the M-band (Fig. 1). Decades of research have shed light on its functions, structure, binding partners and post-translational modifications, for reviews see e.g. Koser et al. (2019), Kruger and Linke (2011), Lange et al. (2006), Zacharchenko et al. (2015). In this review, we will discuss the genetics of titin with respect to cardiac disease and how vertebrate animal and cellular models provide insight into the pathomechanisms related to titin variants. The equally important topic of the role of titin in skeletal muscle disease has been discussed elsewhere (Ottenheijm and Granzier 2010; Savarese et al. 2016).

\section{Identification of cardiomyopathy-associated variants in titin}

The enormous size of the human titin gene (TTN, 363 exons) makes it difficult, expensive and time-consuming to interrogate by conventional mutation screening approaches such as 

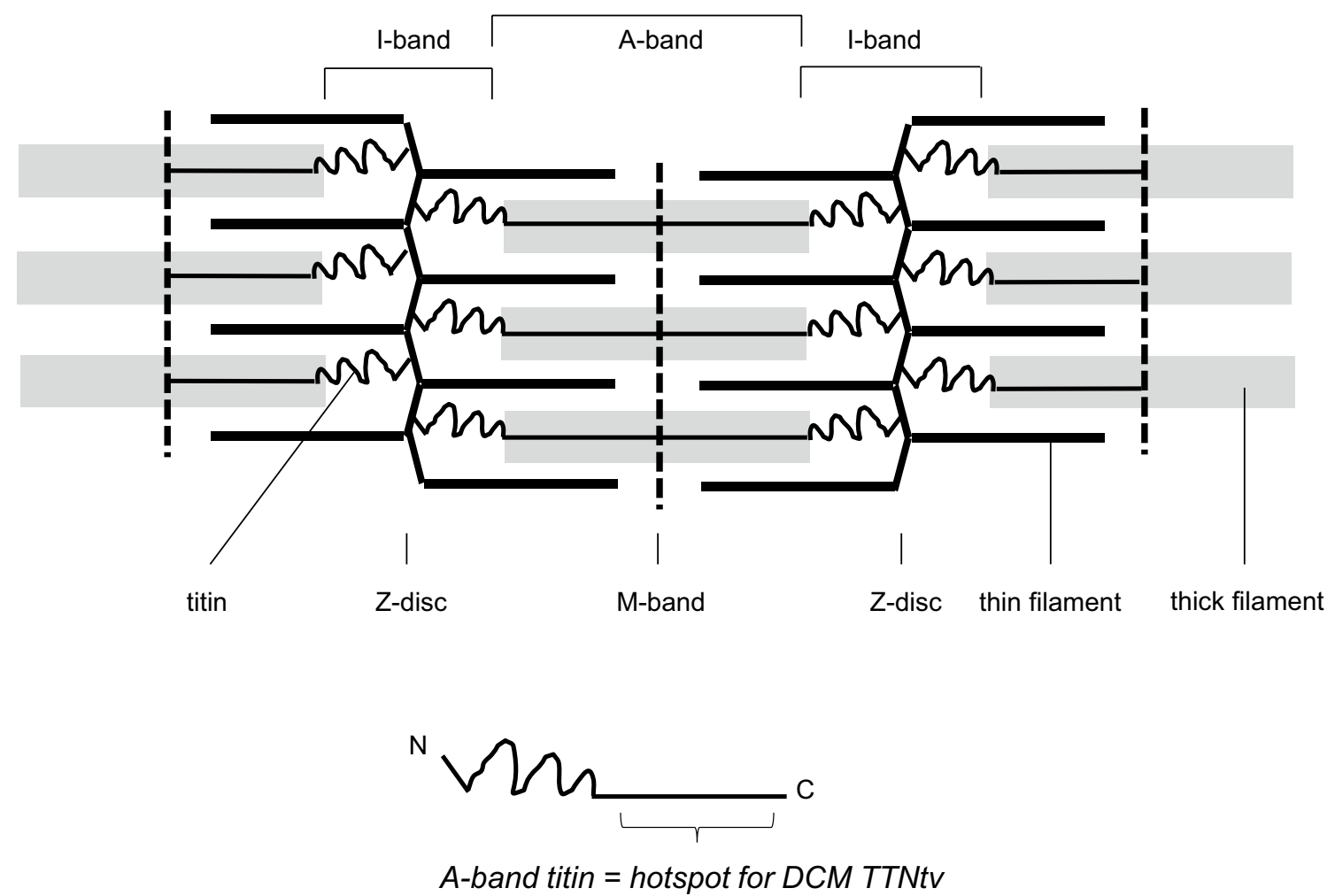

Fig. 1 Schematic drawing of the sarcomere. Each titin molecule (black) spans half the sarcomere from the Z-disc to the M-band, linking thin filaments (bold black) and thick filaments (grey). The positions of I-band and A-band are also indicated. Bottom: The aminoterminus $(\mathrm{N})$ and the carboxy-terminus (C) of an individual titin

Sanger sequencing. Additional complicating factors include (i) alternative splicing, generating various differentially expressed isoforms in tissues and (ii) that a large portion of TTN consists of repetitive tandem Immunoglobulin-like encoding sequences and that variants in these regions may have very little effect on TTN length and function (Greaser 2009).

In 1999, the TTN locus (2q31) was associated with familial autosomal dominant dilated cardiomyopathy (DCM) for the first time (Siu et al. 1999). In the same year, Satoh and colleagues reported the first TTN single-base-pair missense variant in hypertrophic cardiomyopathy (HCM) (Satoh et al. 1999).

Advancement of next-generation sequencing technologies in the last decades has made genetic analyses of TTN more accessible: it can now be interrogated as part of screening for the whole genome, the whole exome or targeted panel of genes in a rapid and cost-effective manner (Norton et al. 2012). In a landmark study in 2012, Herman et al. conducted a genetic analysis of a large number of DCM, HCM and control cases and reported that $T T N$ truncating variants (TTNtv, i.e. variants leading to a premature stop codon in $T T N$ ) are a common cause of DCM, occurring in approximately $25 \%$ molecule are marked (bottom of figure). The A-band portion of titin as a hotspot for DCM-causing variants is indicated. For more detailed schemes of titin domain structure please see Bang et al. (2001), Lange et al. (2006)

of familial and $18 \%$ of sporadic cases, but only present in $1 \%$ of HCM cases. Spatial distribution of variants found in HCM subjects and controls was distinct from that of DCM subjects. The DCM variants were predominantly located in the A-band region. This data has been integrated with RNA sequencing (RNAseq) data from diseased left ventricular samples to help understanding the role of TTNtv (Roberts et al. 2015). This study also determined the mean usage of each TTN exon, denoted as the proportion of transcripts that incorporate each exon (percentage spliced in, PSI) and they estimated that $50 \%$ of the TTNtv identified in healthy controls occur in low PSI exons. Therefore, variants located in high PSI exons, including both N2BA and N2B isoforms, are strongly enriched in DCM patients versus controls. The penetrance of TTNtv in DCM appears to be strongly correlated with its position; distal I-band and all A-band variants show larger odd ratios compared to very proximal or distal ones (Schafer et al. 2017).

TTNtv may cause the generation of short truncated TTN isoforms unable to span between the M-line and the Z-disc, or normal-sized TTN isoforms that attach poorly to the Z-disc (Neiva-Sousa et al. 2015). In either case, the presence 
of abnormal TTN isoforms in the cardiomyocytes results in poorly contractile sarcomeres or may lead to DCM onset.

TTNtv have also be identified in the general population. From analyses of over 4500 control subjects TTNtv have been identified in $1.6 \%$ in individuals of African descent and $1.5 \%$ in individuals of European descent (Roberts et al. 2015). Two different independent studies estimated that $3 \%$ of the population carries a TTNtv allele (Golbus et al. 2012; Herman et al. 2012). In a recent study, a similar frequency of TTNtv ( 2\%) was estimated in a cohort of Chinese HCM patients and one of healthy Chinese controls (Zhang et al. 2017). This study showed for the first time that TTNtv increased the risk of cardiovascular death in patients with HCM and therefore they assigned a role to TTNtv in the phenotypic expression of the disease.

While the interpretation of TTNtv remains difficult due to the high frequency of these variants across normal populations and the lack of penetrance, the situation is far more complex for TTN missense variants, which have been identified in patients affected with different cardiac conditions, such as DCM, HCM, arrhythmogenic cardiomyopathy, restrictive cardiomyopathy and left ventricular non-compaction cardiomyopathy (Arimura et al. 2009; Gerull et al. 2002; Hastings et al. 2016; Itoh-Satoh et al. 2002; Matsumoto et al. 2005; Peled et al. 2014; Satoh et al. 1999; Taylor et al. 2011). However, it is likely that most of them act as 'benign' variants and do not have a role in the development of disease (Begay et al. 2015). In support, a comprehensive study of 530 DCM patients found no enrichment of missense or in-frame small insertions/deletions in TTN when compared to large population cohorts (Akinrinade et al. 2019). Nevertheless, for a rare subset of TTN missense variants, there is clear evidence for their role in the pathogenesis of cardiomyopathies, based on co-segregation studies in large families and in vivo disease models, e.g. Gerull et al. (2002), Hastings et al. (2016).

Although TTN plays a major role in the onset of DCM, due to its complexity, it is currently excluded from the list of genes to be reported as secondary findings by the American College of Medical Genetics (Kalia et al. 2017). When identified in patients, variants in $T T N$, especially missense ones, are often ignored in the first instance. To distinguish the rare disease-relevant variants from the majority of benign ones is a major, still unresolved challenge in clinical practice. Databases and bioinformatics tools are developed to help with the evaluation and interpretation of TTN variants (Table 1) and novel cellular model systems (see below) may help to address this issue experimentally in the future, as it has already been demonstrated for other cardiomyopathy genes (Ma et al. 2018).

\section{Insights into titin-related cardiomyopathy studying human hearts}

Given the scarcity of human cardiac tissue for research purposes, especially from healthy control individuals, it is not surprising that only few studies have looked at molecular changes in the hearts of cardiomyopathy patients carrying titin variants (Roberts et al. 2015; Vikhorev et al. 2017). The main outcome of these studies is that no truncated titin proteins could be detected in individuals with TTNtv and that overall levels of titin transcript and protein are normal. Likewise, the isoform ratio N2B/N2BA is not altered. These findings argue against a haplo-insufficiency mode of action, yet support a dominant negative mode of action, but not through a poisonous-peptide mechanism. RNAseq data from 34 DCM patients with TTNtv identified alterations in mitochondrial function and fibrosis as molecular hallmarks of the disease (Verdonschot et al. 2018); however, these are unspecific features of many cardiomyopathies (Schirone et al. 2017).

\section{Modelling titin variants in animal models}

Whole organism models are valuable tools to study the consequences of titin variants on the hearts. Important insights on titin function were gained from studying shorter titin-like proteins in invertebrates, such as twitchin in C. elegans and kettin in Drosophila; for reviews see Benian et al. (1999), Bullard et al. (2005), Ferrara et al. (2005). Zebrafish and mouse are the most commonly used animals to study cardiac functions of titin, but rat models and even a dog model also exist.

Table 1 Online resources to evaluate titin variants

\begin{tabular}{lll}
\hline Name & Link & References \\
\hline Titin variants in dilated cardiomyopathy & https://www.cardiodb.org/titin/ & Roberts et al. (2015) \\
Leiden open variation database & https://databases.lovd.nl/shared/genes/TTN & Fokkema et al. (2005) \\
Genome aggregation database & https://gnomad.broadinstitute.org/gene/ENSG00000155657 & Karczewski et al. (2019) \\
Titindb & http://fraternalilab.kcl.ac.uk/TITINdb/ & Laddach et al. (2017) \\
\hline
\end{tabular}




\section{Zebrafish models}

Traditionally, zebrafish is a popular model organisms because of the ease of genetic modulation via random mutagenesis or morpholino oligomers, its short reproductive span and the transparent heart of its embryos, facilitating in vivo imaging (North and Zon 2003).

The pickwick mutant line is characterised by reduced contractility of its heart and poor myofibrillar maturation without mature sarcomeres (Xu et al. 2002), it is embryonic lethal. A homozygous single base pair change in the N2B region of titin, introducing a stop codon, was found to be the underlying genetic cause. It soon became apparent that there are two titin orthologs in zebrafish (ttna and ttnb) and that both the N2B-exon and N2A-exon containing isoforms of ttna are required for sarcomere assembly in the heart, while ttnb is dispensable for cardiac contractility (Seeley et al. 2007). An internal promotor, giving rise to the Cronos transcript, a C-terminal titin isoform, may explain why the position of truncation modulates disease severity, as was shown for skeletal muscle (Zou et al. 2015). However, there is still controversy about the evolutionary conversation and relevance of the Cronos transcript (Deo 2016; Shih et al. 2016).

Moreover, the zebrafish model has provided insight into the increased risk of atrial fibrillation in individuals carrying TTNtv (Ahlberg et al. 2018). A heterozygous TTNtv resulted in sarcomere defects in both ventricular and atrial chamber in the adult fish, as well as fibrosis and increased PR interval. These pathological changes may form a substrate for atrial fibrillation.

\section{Rodent and large animal models}

Mice are by far the most established model organism to study gene function and its perturbation through genetic variants. As the global knock-out of titin is embryonic lethal (Radke et al. 2019), studies deleted important regions of the protein, such as the M-band portion (Gotthardt et al. 2003; Radke et al. 2019), the N2B region (Radke et al. 2007) and the PEVK region (Granzier et al. 2009), to understand the contributions of these regions to titin's functions. All manipulations lead to disturbances in cardiac function, however the exact phenotype (DCM, hypertrophy or atrophy) depends on the deleted region.

Gramlich et al. (2009) introduced the human DCM-causing TTNtv c. 43628insAT (Gerull et al. 2002) into a mouse model. In the homozygous setting the variant is embryonic lethal, while heterozygous mice have no overt phenotype. However, upon chronic challenge with angiotensin or upon pressure overload via trans-aortic constriction for 2 weeks, the heterozygous mice developed features of DCM, namely systolic dysfunction and dilatation, while wild-type mice undergoing the same treatment had preserved cardiac dimensions and function. Also, TTNtv mice had increased levels of fibrosis upon both challenges (Gramlich et al. 2009; Zhou et al. 2015). The angiotensin-induced phenotype of the mouse model could be rescued by antisense-mediated exon skipping, removing the premature stop from the transcript (Gramlich et al. 2015). Another successful therapeutic approach to improve cardiac function in these mice was inhibition of miR-208b, a micro-RNA shown to be upregulated in the model (Zhou et al. 2017).

Rat models have been generated carrying TTNtv in either the Z-disc or the A-band portion of titin (Schafer et al. 2017). Like their mouse counterparts, homozygous rats were not viable. Young heterozygous rats ( $<8$ months) have preserved cardiac function, but showed signs of concentric remodelling. In old rats ( $>1$ year), mild systolic dysfunction, not quite reaching statistical significance in all parameters, was observed, irrespective of the position of the variant. The main value of the models is that ribo-sequencing and RNAseq data revealed that $T T N$ transcripts with an A-band TTNtv were transcribed and translated as far as the premature nonsense codon, where the translation stops; whereas the premature stop codon of the TTNtv in the Z-disc did not prevent translation downstream. Regardless the position of the variant within the protein, TTNtv lead to nonsensemediated degradation of the mutant allele and a signature of perturbed cardiac metabolism but they result in different translational footprints (Schafer et al. 2017).

A further study subjected the rat model with the TTNtv at the Z-disc to pressure overload by trans-aortic constriction. This resulted in mild diastolic dysfunction, increased fibrosis and reduced capillary density. Additionally, more apoptosis was observed in these mice (Ye et al. 2018). Finally, a more detailed molecular analysis of both rat models revealed impaired autophagy and mitochondrial defects leading to reactive oxygen species production and increased acetylation of mitochondrial proteins as disease mechanisms (Zhou et al. 2019). The study further demonstrated that mTOR inhibitor rapamycin can rescue the autophagy defects in these rat models, leading to the speculation that this could be an intervention to ameliorate ventricular remodelling associated with TTNtv.

Furthermore, a dog model of titin missense variant exists (Meurs et al. 2019), this homozygous or heterozygous missense change in immunoglobulin domain I71 in the N2BA isoform was identified in a Doberman pinscher dog pedigree with familial DCM and a high incidence of sudden cardiac death. 


\section{Modelling titin variants in induced pluripotent stem cell derived cardiomyocytes}

Major cellular reprogramming breakthroughs now allow the reversal of terminally differentiated human cells (e.g. foreskin or skin fibroblasts, white blood cells) into pluripotency using reprogramming factors (Oct4, Sox 2, Klf4, and c-myc) (Takahashi and Yamanaka 2006). Directed differentiation of human-induced pluripotent stem cells (iPSCs) into cardiomyocytes (iPSC-CMs) can be achieved by manipulation of Wnt signalling (Lian et al. 2012). iPSC-CMs can provide a long-term human in vitro model to investigate titin variants and their role in cardiomyopathy; they have the potential to mimic human pathophysiology as they are not affected by species-related physiological differences (such as heart rate and ion channel setup) between humans and rodents. However, foetal characteristics and immaturity are major drawbacks of iPSC-CMs, for review of this subject see Jiang et al. (2018), Yang et al. (2014). Tissue engineering techniques such as cardiac microtissue, engineered heart tissue and engineered human myocardium are 3D culturing techniques that enable more mature phenotypes of iPSC-CMs, e.g. in respect to sarcomere organisation (Giacomelli et al. 2017; Hansen et al. 2010; Tiburcy et al. 2017). Alternatively, nanopatterning on substrates of physiological stiffness (Ribeiro

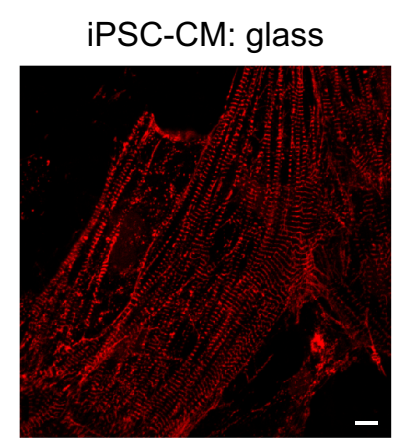

iPSC-CM: nano-patterned

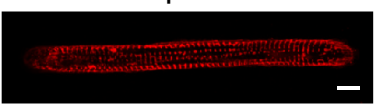

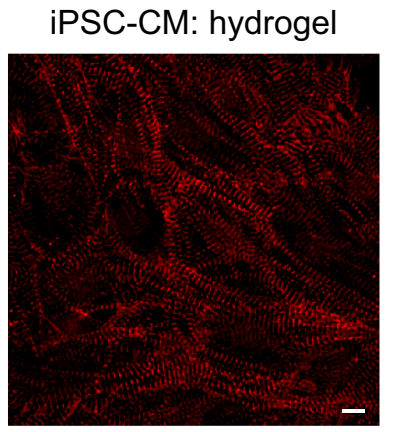

adult mouse cardiomyocyte

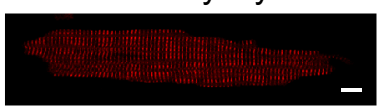

Fig. 2 Immature phenotype of iPSC-CM in conventional 2D cultures: Normal Kolf2 iPSC (Streeter et al. 2017) were differentiated into iPSC-CMs using standard methods (Burridge et al. 2015) and plated on day 20 on different substrates: matrigel coated glass (top left), matrigel coated hydrogel (top right, $7 \mathrm{kPa}$ ) and nanopatterned matrigel coated hydrogel (bottom left, see Ribeiro et al. (2015)). 30 days after induction of differentiation, cells were fixed and sarcomeric structures visualised with an antibody direct against alpha-actinin (EA53, Sigma). For comparison, an isolated mouse cardiomyocyte stained with the same antibody is shown (bottom right). Scale bars represent 10 microns et al. 2015), scaffolds (Blazeski et al. 2019) or bioactive lipids (Sharma et al. 2018) have been shown to improve iPSC-CM maturation. However, iPSC-CM are unlikely to reach full characteristics of adult cardiomyocytes morphologically or physiologically (Fig. 2).

iPSC-CMs are particularly powerful when combined with genome-editing techniques such as Clustered regularly interspaced short palindromic repeat (CRISPR)/CRISPRassociated protein 9 (Cas9) or Transcription activator-like effector nucleases (Chen et al. 2015; Ding et al. 2013). In principle, there are two approaches to study the consequences of variants e.g. in titin: patient-derived iPSC-CMs carrying the disease-associated variant are compared to iPSC-CMs from healthy control individuals (ideally close, unaffected family members) and/or an iPSC line in which the disease-associated variant is reverted to the wildtype allele via genome-editing. An alternative approach is to introduce a disease-associated variant into iPSC derived from a normal individual and using isogenic un-edited or control-edited iPSC as controls. The advantage of the latter approach is that both hetero- and homozygous variants can be generated. Examples of both approaches will now be discussed:

iPSC-CMs derived from DCM patients carrying A-band TTNtv (Ser14450fsX and Ser19628IfsX) exhibited a marked reduction in contraction amplitude, alterations in calcium transients and sarcomeric structural disruption, with less iPSC-CM displaying fully organised sarcomeres compared to iPSC-CM from a healthy control individual (Gramlich et al. 2015; Schick et al. 2018). Rescue by skipping the affected exon with antisense oligonucleotides resulted in improved myofibril assembly and stability as well as in a normalised gene expression profile, similar to the functional rescue observed in a corresponding mouse model (see above) (Gramlich et al. 2015).

Hinson et al. (2015) carried out an investigation into the penetrance of two TTNtv (Ala22352fsX and Pro22582fsX) and one missense variant (Trp976Arg) in the A-band and Z/I-band junction of the sarcomere respectively using both patient-derived iPSC-CMs and genome-edited iPSC-CMs, both in 2D cultures and cardiac microtissue. Both TTNtv lines displayed significant reductions in contractile forces compared to wildtype iPSC-CMs although I-band TTNtv did not result in the same pronounced pathogenic phenotypes inferring a reduced penetrance (Hinson et al. 2015). A further study of an A-band TTNtv showed that the resultant shortened titin also displayed impaired contractile performance, poor sarcomere assembly and myosin mechanical force (Chopra et al. 2018).

Taken together, the small number of studies of DCMassociated titin variants in iPSC-CMs so far have revealed reduced contractility and impaired sarcomere assembly as contributors to the disease. 


\section{New methodological developments: opportunities to advance the use of IPSC-CM for studying titin variants}

Functional assessment of contractility is still an issue in iPSC-CM, as established methods used for adult mouse cardiomyocytes, which track changes of the visible striation of highly organised sarcomeres, fail in the less organised iPSC-CM. However, recent advancements of open-source contractility analysis software tools such as MuscleMotion and SarcTrack will help to shed light on the role of titin variants in cardiac contractility: MuscleMotion is an ImageJ plug-in allowing the label-free quantification of contraction of e.g. individual iPSC-CMs, 2D monolayers or engineered heart tissue based on analysing changes in pixel intensity relative to a reference frame (Sala et al. 2018). SarckTrack is a recent MatLab contractility software which allows hundreds of individual fluorescently-labelled sarcomeres in iPSC-CM to be analysed in each cell (Toepfer et al. 2019).

Moreover, recent diversifications of the CRISPR/Cas9 toolbox open new avenues to manipulate iPSC-CMs: its traditional application is in genome-editing, utilising nonhomologous end joining or homologous directed repair (reviewed in Devkota (2018), Singh et al. (2017)). The former is commonly used to generate null alleles via small insertion/deletion disrupting the reading frame (Chopra et al. 2018), the latter is used to introduce fusion tags e.g. a green fluorescent protein titin fusion to visualise sarcomeres (Toepfer et al. 2019) or site-specific variants, e.g. TTNtv (Hinson et al. 2015).

The specificity of CRISPR is also captured as a sitespecific molecular localiser. Different functional molecules can be tethered with catalytically deficient Cas9 (dCas9) and guided to the target locus without genetic perturbation (Knott and Doudna 2018; Larson et al. 2013). Firstly, the CRISPR activation system (CRISPRa) recruits positive transcription regulators to the promoter or proximal enhancer regions and thereby drives gene expression (Hilton et al. 2015; Konermann et al. 2015; Zhang et al. 2015). This technology wins over the conventional overexpression systems, which depend on a random integration of the expression vectors, as it does not affect the host's DNA sequence. Various modified versions of this gene regulation tool have been developed to include different effectors, or in combination, to maximise the fold activation (Chavez et al. 2016; Hilton et al. 2015). Modelling disease systems with distinguished expression profiles and governing key transcription factor expression to direct differentiation (Weltner et al. 2018) are promising applications of CRISPRa.

On the contrary, CRISPR interference (CRISPRi), blocks transcriptional activity by a steric hindrance between dCas 9 and the transcriptional machinery (Larson et al. 2013) or by the actions of tethered transcription repressors (Parsi et al. 2017). Compared to existing gene knockdown methods owing to various advantages: CRISPRi is more effective in gene silencing as it acts directly at the transcriptional status, while RNA interference modulates the expression at mRNA level (Agrawal et al. 2003). Moreover, RNA interference targets are limited to cytosolic mRNAs but CRISPRi holds a broader target repertoire, including coding and non-coding sequences (Gilbert et al. 2014). The modularity of CRISPR system makes it easy to create guide RNA libraries. Lastly, the repression activity can be fine-tuned by changing the target region or by probing multiple sites simultaneouslyCRISPRi can even be switched on or off using inducible CRISPRi-KRAB (Parsi et al. 2017) if time-dependent study is needed for example to dissect developmental phases.

In summary, the CRISPR/Cas9 system provides exciting tools beyond conventional genome-editing to study e.g. the consequences of titin variants in the cardiac system.

\section{Conclusion}

While a causal role for TTNtv in DCM is now well established, the underlying disease pathways are still not fully understood. Animal models have highlighted a crucial role of titin for the integrity of the heart and have helped to gain insight into aspects of titin's function, such as sarcomere assembly and signalling and their perturbations by titin variants. However, animal models are limited by species-related differences in morphology and physiology to humans. As an alternative, studies of human cardiac tissue can also provide insights into the patho-mechanisms of titin variants, but such tissue is rarely available in sufficient quality and quantity. Therefore iPSC-CM emerge as a novel model system to study titin-related cardiomyopathy. These cells allow mimicking human genetic disease in a human cardiac cellular model. While still in its infancy and currently plagued by the issue of immaturity, it provides exciting approaches to study the role of titin variants in cardiomyopathies and we are looking forward to future developments in the field.

Acknowledgements We would like to thank Hoadi Wu and Timon Seeger (Stanford University) for help with culturing of iPSC-CM on various substrates. KG is supported by a British Heart Foundation (BHF) Grant (FS/12/40/29712). KG and GP acknowledge support from the BHF Centre of Research Excellence, Oxford (RE/13/1/30181). KG and AJ acknowledge support from the Wellcome Trust $(201543 / \mathrm{B} / 16 / \mathrm{Z})$. NS is supported by a scholarship from the Royal Thai government.

Open Access This article is distributed under the terms of the Creative Commons Attribution 4.0 International License (http://creativeco mmons.org/licenses/by/4.0/), which permits unrestricted use, distribution, and reproduction in any medium, provided you give appropriate 
credit to the original author(s) and the source, provide a link to the Creative Commons license, and indicate if changes were made.

\section{References}

Agrawal N, Dasaradhi PV, Mohmmed A, Malhotra P, Bhatnagar RK, Mukherjee SK (2003) RNA interference: biology, mechanism, and applications. Microbiol Mol Biol Rev 67:657-685

Ahlberg G, Refsgaard L, Lundegaard PR et al (2018) Rare truncating variants in the sarcomeric protein titin associate with familial and early-onset atrial fibrillation. Nat Commun 9:4316. https://doi. org/10.1038/s41467-018-06618-y

Akinrinade O, Helio T, Lekanne Deprez RH et al (2019) Relevance of titin missense and non-frameshifting insertions/deletions variants in dilated cardiomyopathy. Sci Rep 9:4093. https://doi. org/10.1038/s41598-019-39911-x

Arimura T, Bos JM, Sato A et al (2009) Cardiac ankyrin repeat protein gene (ANKRD1) mutations in hypertrophic cardiomyopathy. J Am Coll Cardiol 54:334-342. https://doi.org/10.1016/j. jacc.2008.12.082

Bang ML, Centner T, Fornoff F et al (2001) The complete gene sequence of titin, expression of an unusual approximately 700$\mathrm{kDa}$ titin isoform, and its interaction with obscurin identify a novel Z-line to I-band linking system. Circ Res 89:1065-1072

Begay RL, Graw S, Sinagra G et al (2015) Role of titin missense variants in dilated cardiomyopathy. J Am Heart Assoc. https://doi. org/10.1161/jaha.115.002645

Benian GM, Ayme-Southgate A, Tinley TL (1999) The genetics and molecular biology of the titin/connectin-like proteins of invertebrates. Rev Physiol Biochem Pharmacol 138:235-268

Blazeski A, Lowenthal J, Wang Y et al (2019) Engineered heart slice model of arrhythmogenic cardiomyopathy using Plakophilin-2 mutant myocytes. Tissue Eng Part A. https://doi.org/10.1089/ ten.tea.2018.0272

Bullard B, Burkart C, Labeit S, Leonard K (2005) The function of elastic proteins in the oscillatory contraction of insect flight muscle. J Muscle Res Cell Motil 26:479-485. https://doi.org/10.1007/ s10974-005-9032-7

Burridge PW, Holmstrom A, Wu JC (2015) Chemically defined culture and cardiomyocyte differentiation of human pluripotent stem cells. Curr Protoc Hum Genet. https://doi.org/10.1002/04711 42905.hg2103s 87

Chavez A, Tuttle M, Pruitt BW et al (2016) Comparison of Cas9 activators in multiple species. Nat Methods 13:563-567. https://doi. org/10.1038/nmeth.3871

Chen YJ, Cao JY, Xiong M et al (2015) Engineering human stem cell lines with inducible gene knockout using CRISPR/Cas9. Cell Stem Cell 17:233-244. https://doi.org/10.1016/j.stem.2015.06.001

Chopra A, Kutys ML, Zhang KH et al (2018) Force generation via beta-cardiac myosin, titin, and alpha-actinin drives cardiac sarcomere assembly from cell-matrix adhesions. Dev Cell. https:// doi.org/10.1016/j.devcel.2017.12.012

Deo RC (2016) Alternative splicing, internal promoter, nonsense-mediated decay, or all three: explaining the distribution of truncation variants in titin. Circ Cardiovasc Genet 9:419-425. https://doi. org/10.1161/CIRCGENETICS.116.001513

Devkota S (2018) The road less traveled: strategies to enhance the frequency of homology-directed repair (HDR) for increased efficiency of CRISPR/Cas-mediated transgenesis. BMB Rep 51:437-443

Ding QR, Regan SN, Xia YL, Oostrom LA, Cowan CA, Musunuru K (2013) Enhanced efficiency of human pluripotent stem cell genome editing through replacing TALENs with CRISPRs. Cell Stem Cell 12:393-394. https://doi.org/10.1016/j.stem.2013.03.006

Ferrara TM, Flaherty DB, Benian GM (2005) Titin/connectin-related proteins in C. elegans: a review and new findings. J Muscle Res Cell Motil 26:435-447. https://doi.org/10.1007/s 1097 4-005-9027-4

Fokkema IF, den Dunnen JT, Taschner PE (2005) LOVD: easy creation of a locus-specific sequence variation database using an "LSDB-in-a-box" approach. Hum Mutat 26:63-68. https://doi. org/10.1002/humu.20201

Gerull B, Gramlich M, Atherton J et al (2002) Mutations of TTN, encoding the giant muscle filament titin, cause familial dilated cardiomyopathy. Nat Genet 30:201-204. https://doi.org/10.1038/ ng815

Giacomelli E, Bellin M, Sala L et al (2017) Three-dimensional cardiac microtissues composed of cardiomyocytes and endothelial cells co-differentiated from human pluripotent stem cells. Development 144:1008-1017. https://doi.org/10.1242/dev.143438

Gilbert LA, Horlbeck MA, Adamson B et al (2014) Genome-Scale CRISPR-mediated control of gene repression and activation. Cell 159:647-661. https://doi.org/10.1016/j.cell.2014.09.029

Golbus JR, Puckelwartz MJ, Fahrenbach JP, Dellefave-Castillo LM, Wolfgeher D, McNally EM (2012) Population-based variation in cardiomyopathy genes. Circ Cardiovasc Genet 5:391-399. https ://doi.org/10.1161/CIRCGENETICS.112.962928

Gotthardt M, Hammer RE, Hubner N et al (2003) Conditional expression of mutant $\mathrm{M}$-line titins results in cardiomyopathy with altered sarcomere structure. J Biol Chem 278:6059-6065. https://doi. org/10.1074/jbc.M211723200

Gramlich M, Michely B, Krohne C et al (2009) Stress-induced dilated cardiomyopathy in a knock-in mouse model mimicking human titin-based disease. J Mol Cell Cardiol 47:352-358. https://doi. org/10.1016/j.yjmcc.2009.04.014

Gramlich M, Pane LS, Zhou QF et al (2015) Antisense-mediated exon skipping: a therapeutic strategy for titin-based dilated cardiomyopathy. Embo Mol Med 7:562-576. https://doi.org/10.15252 /emmm.201505047

Granzier HL, Radke MH, Peng J et al (2009) Truncation of titin's elastic PEVK region leads to cardiomyopathy with diastolic dysfunction. Circ Res 105:557-564. https://doi.org/10.1161/CIRCR ESAHA.109.200964

Greaser ML (2009) Stressing the giant: a new approach to understanding dilated cardiomyopathy. J Mol Cell Cardiol 47:347-349. https ://doi.org/10.1016/j.yjmcc.2009.06.011

Hansen A, Eder A, Bonstrup M et al (2010) Development of a drug screening platform based on engineered heart tissue. Circ Res 107:35-U70. https://doi.org/10.1161/circresaha.109.211458

Hastings R, de Villiers CP, Hooper C et al (2016) Combination of whole genome sequencing, linkage, and functional studies implicates a missense mutation in titin as a cause of autosomal dominant cardiomyopathy with features of left ventricular noncompaction. Circ Cardiovasc Genet 9:426-435. https://doi.org/10.1161/ CIRCGENETICS.116.001431

Herman DS, Lam L, Taylor MR et al (2012) Truncations of titin causing dilated cardiomyopathy. N Engl J Med 366:619-628. https:// doi.org/10.1056/NEJMoa1110186

Hilton IB, D'Ippolito AM, Vockley CM et al (2015) Epigenome editing by a CRISPR-Cas9-based acetyltransferase activates genes from promoters and enhancers. Nat Biotechnol 33:510-517. https://doi. org/10.1038/nbt.3199

Hinson JT, Chopra A, Nafissi N et al (2015) Titin mutations in iPS cells define sarcomere insufficiency as a cause of dilated cardiomyopathy. Science 349:982-986. https://doi.org/10.1126/scien ce.aaa5458 
Itoh-Satoh M, Hayashi T, Nishi H et al (2002) Titin mutations as the molecular basis for dilated cardiomyopathy. Biochem Biophys Res Commun 291:385-393. https://doi.org/10.1006/bbrc.2002.6448

Jiang Y, Park P, Hong SM, Ban K (2018) Maturation of cardiomyocytes derived from human pluripotent stem cells: current strategies and limitations. Mol Cells 41:613-621. https://doi.org/10.14348 /molcells.2018.0143

Kalia SS, Adelman K, Bale SJ et al (2017) Recommendations for reporting of secondary findings in clinical exome and genome sequencing, 2016 update (ACMG SF v2.0): a policy statement of the American College of Medical Genetics and Genomics. Genet Med 19:249-255. https://doi.org/10.1038/gim.2016.190

Karczewski KJ, Francioli LC, Tiao G et al (2019) Variation across 141,456 human exomes and genomes reveals the spectrum of loss-of-function intolerance across human protein-coding genes. BioRxiv:531210

Knott GJ, Doudna JA (2018) CRISPR-Cas guides the future of genetic engineering. Science 361:866-869. https://doi.org/10.1126/scien ce.aat5011

Konermann S, Brigham MD, Trevino AE et al (2015) Genome-scale transcriptional activation by an engineered CRISPR-Cas9 complex. Nature 517:583-588. https://doi.org/10.1038/nature14136

Koser F, Loescher C, Linke WA (2019) Posttranslational modifications of titin from cardiac muscle: how, where, and what for? FEBS J. https://doi.org/10.1111/febs.14854

Kruger M, Linke WA (2011) The giant protein titin: a regulatory node that integrates myocyte signaling pathways. J Biol Chem 286:9905-9912. https://doi.org/10.1074/jbc.R110.173260

Laddach A, Gautel M, Fraternali F (2017) TITINdb-a computational tool to assess titin's role as a disease gene. Bioinformatics 33:3482-3485. https://doi.org/10.1093/bioinformatics/btx424

Lange S, Ehler E, Gautel M (2006) From A to Z and back? Multicompartment proteins in the sarcomere. Trends Cell Biol 16:11-18. https://doi.org/10.1016/j.tcb.2005.11.007

Larson MH, Gilbert LA, Wang X, Lim WA, Weissman JS, Qi LS (2013) CRISPR interference (CRISPRi) for sequence-specific control of gene expression. Nat Protoc 8:2180-2196. https://doi. org/10.1038/nprot.2013.132

Lian X, Hsiao C, Wilson G et al (2012) Robust cardiomyocyte differentiation from human pluripotent stem cells via temporal modulation of canonical Wnt signaling. Proc Natl Acad Sci USA 109:E1848-E1857. https://doi.org/10.1073/pnas.1200250109

Ma N, Zhang JZ, Itzhaki I et al (2018) Determining the pathogenicity of a genomic variant of uncertain significance using CRISPR/Cas9 and human-induced pluripotent stem cells. Circulation 138:2666-2681. https://doi.org/10.1161/CIRCULATIO NAHA.117.032273

Maruyama K (1976) Connectin, an elastic protein from myofibrils. J Biochem 80:405-407. https://doi.org/10.1093/oxfordjournals. jbchem.a131291

Matsumoto Y, Hayashi T, Inagaki N et al (2005) Functional analysis of titin/connectin N2-B mutations found in cardiomyopathy. J Muscle Res Cell Motil 26:367-374. https://doi.org/10.1007/ s10974-005-9018-5

Meurs KM, Friedenberg SG, Kolb J et al (2019) A missense variant in the titin gene in Doberman pinscher dogs with familial dilated cardiomyopathy and sudden cardiac death. Hum Genet. https:// doi.org/10.1007/s00439-019-01973-2

Neiva-Sousa M, Almeida-Coelho J, Falcao-Pires I, Leite-Moreira AF (2015) Titin mutations: the fall of Goliath. Heart Fail Rev 20:579_ 588. https://doi.org/10.1007/s10741-015-9495-6

North TE, Zon LI (2003) Modeling human hematopoietic and cardiovascular diseases in zebrafish. Dev Dyn 228:568-583. https://doi. org/10.1002/dvdy. 10393
Norton N, Li D, Hershberger RE (2012) Next-generation sequencing to identify genetic causes of cardiomyopathies. Curr Opin Cardiol 27:214-220. https://doi.org/10.1097/HCO.0b013e328352207e

Ottenheijm CA, Granzier H (2010) Role of titin in skeletal muscle function and disease. Adv Exp Med Biol 682:105-122. https:// doi.org/10.1007/978-1-4419-6366-6_6

Parsi KM, Hennessy E, Kearns N, Maehr R (2017) Using an inducible CRISPR-dCas9-KRAB effector system to dissect transcriptional regulation in human embryonic stem cells. Methods Mol Biol 1507:221-233. https://doi.org/10.1007/978-1-4939-6518-2_16

Peled Y, Gramlich M, Yoskovitz G et al (2014) Titin mutation in familial restrictive cardiomyopathy. Int J Cardiol 171:24-30. https:// doi.org/10.1016/j.ijcard.2013.11.037

Radke MH, Peng J, Wu Y et al (2007) Targeted deletion of titin N2B region leads to diastolic dysfunction and cardiac atrophy. Proc Natl Acad Sci USA 104:3444-3449. https://doi.org/10.1073/ pnas.0608543104

Radke MH, Polack C, Methawasin M, Fink C, Granzier HL, Gotthardt M (2019) Deleting full length titin versus the titin M-band region leads to differential mechanosignaling and cardiac phenotypes. Circulation 139:1813-1827. https://doi.org/10.1161/CIRCU LATIONAHA.118.037588

Ribeiro AJ, Ang YS, Fu JD et al (2015) Contractility of single cardiomyocytes differentiated from pluripotent stem cells depends on physiological shape and substrate stiffness. Proc Natl Acad Sci USA 112:12705-12710. https://doi.org/10.1073/pnas.1508073112

Roberts AM, Ware JS, Herman DS et al (2015) Integrated allelic, transcriptional, and phenomic dissection of the cardiac effects of titin truncations in health and disease. Sci Transl Med. https://doi. org/10.1126/scitranslmed.3010134

Sala L, van Meer BJ, Tertoolen LGJ et al (2018) MUSCLEMOTION: a versatile open software tool to quantify cardiomyocyte and cardiac muscle contraction in vitro and in vivo. Circ Res 122:e5-e16. https://doi.org/10.1161/circresaha.117.312067

Satoh M, Takahashi M, Sakamoto T, Hiroe M, Marumo F, Kimura A (1999) Structural analysis of the titin gene in hypertrophic cardiomyopathy: identification of a novel disease gene. Biochem Biophys Res Commun 262:411-417. https://doi.org/10.1006/ bbrc.1999.1221

Savarese M, Sarparanta J, Vihola A, Udd B, Hackman P (2016) Increasing role of titin mutations in neuromuscular disorders. J Neuromuscul Dis 3:293-308. https://doi.org/10.3233/JND-16015 8

Schafer S, de Marvao A, Adami E et al (2017) Titin-truncating variants affect heart function in disease cohorts and the general population. Nat Genet 49:46-53. https://doi.org/10.1038/ng.3719

Schick R, Mekies LN, Shemer Y, Eisen B, Hellas T, Jehuda RB (2018) Functional abnormalities in induced pluripotent stem cell-derived cardiomyocytes generated from titin-mutated patients with dilated cardiomyopathy. PLoS ONE 13:e0205719. https://doi. org/10.1371/journal.pone.0207548

Schirone L, Forte M, Palmerio S et al (2017) A review of the molecular mechanisms underlying the development and progression of cardiac remodeling. Oxid Med Cell Longev 2017:3920195. https ://doi.org/10.1155/2017/3920195

Seeley M, Huang W, Chen Z, Wolff WO, Lin X, Xu X (2007) Depletion of zebrafish titin reduces cardiac contractility by disrupting the assembly of Z-discs and A-bands. Circ Res 100:238-245. https:// doi.org/10.1161/01.RES.0000255758.69821.b5

Sharma A, Zhang Y, Buikema JW et al (2018) Stage-specific effects of bioactive lipids on human iPSC cardiac differentiation and cardiomyocyte proliferation. Sci Rep 8:6618. https://doi.org/10.1038/ s41598-018-24954-3

Shih YH, Dvornikov AV, Zhu P et al (2016) Exon- and contractiondependent functions of titin in sarcomere assembly. Development 143:4713-4722. https://doi.org/10.1242/dev.139246 
Singh V, Braddick D, Dhar PK (2017) Exploring the potential of genome editing CRISPR-Cas9 technology. Gene 599:1-18. https ://doi.org/10.1016/j.gene.2016.11.008

Siu BL, Niimura H, Osborne JA et al (1999) Familial dilated cardiomyopathy locus maps to chromosome 2q31. Circulation 99:1022-1026

Streeter I, Harrison PW, Faulconbridge A et al (2017) The humaninduced pluripotent stem cell initiative-data resources for cellular genetics. Nucleic Acids Res 45:D691-D697. https://doi. org/10.1093/nar/gkw928

Takahashi K, Yamanaka S (2006) Induction of pluripotent stem cells from mouse embryonic and adult fibroblast cultures by defined factors. Cell 126:663-676. https://doi.org/10.1016/j. cell.2006.07.024

Taylor M, Graw S, Sinagra G et al (2011) Genetic variation in titin in arrhythmogenic right ventricular cardiomyopathy-overlap syndromes. Circulation 124:876-885. https://doi.org/10.1161/CIRCU LATIONAHA.110.005405

Tiburcy M, Hudson JE, Balfanz P et al (2017) Defined engineered human myocardium with advanced maturation for applications in heart failure modeling and repair. Circulation. https://doi. org/10.1161/circulationaha.116.024145

Toepfer CN, Sharma A, Cicconet M et al (2019) SarcTrack: an adaptable software tool for efficient large-scale analysis of sarcomere function in hiPSC-cardiomyocytes. Circ Res. https://doi. org/10.1161/circresaha.118.314505

Verdonschot JAJ, Hazebroek MR, Derks KWJ et al (2018) Titin cardiomyopathy leads to altered mitochondrial energetics, increased fibrosis and long-term life-threatening arrhythmias. Eur Heart $\mathbf{J}$ 39:864-873. https://doi.org/10.1093/eurheartj/ehx808

Vikhorev PG, Smoktunowicz N, Munster AB et al (2017) Abnormal contractility in human heart myofibrils from patients with dilated cardiomyopathy due to mutations in TTN and contractile protein genes. Sci Rep 7:14829. https://doi.org/10.1038/s41598-01713675-8

Weltner J, Balboa D, Katayama S et al (2018) Human pluripotent reprogramming with CRISPR activators. Nat Commun 9:2643. https:// doi.org/10.1038/s41467-018-05067-x

Xu X, Meiler SE, Zhong TP et al (2002) Cardiomyopathy in zebrafish due to mutation in an alternatively spliced exon of titin. Nat Genet 30:205-209. https://doi.org/10.1038/ng816

Yang X, Pabon L, Murry CE (2014) Engineering adolescence: maturation of human pluripotent stem cell-derived cardiomyocytes.
Circ Res 114:511-523. https://doi.org/10.1161/CIRCRESAHA .114 .300558

Ye L, Su L, Wang C et al (2018) Truncations of the titin Z-disc predispose to a heart failure with preserved ejection phenotype in the context of pressure overload. PLoS ONE 13:e0201498. https:// doi.org/10.1371/journal.pone.0201498

Zacharchenko T, von Castelmur E, Rigden DJ, Mayans O (2015) Structural advances on titin: towards an atomic understanding of multi-domain functions in myofilament mechanics and scaffolding. Biochem Soc Trans 43:850-855. https://doi.org/10.1042/ BST20150084

Zhang Y, Yin C, Zhang T et al (2015) CRISPR/gRNA-directed synergistic activation mediator (SAM) induces specific, persistent and robust reactivation of the HIV-1 latent reservoirs. Sci Rep 5:16277. https://doi.org/10.1038/srep16277

Zhang C, Zhang H, Wu G et al (2017) Titin-truncating variants increase the risk of cardiovascular death in patients with hypertrophic cardiomyopathy. Can J Cardiol 33:1292-1297. https://doi. org/10.1016/j.cjca.2017.05.020

Zhou Q, Kesteven S, Wu J et al (2015) Pressure overload by transverse aortic constriction induces maladaptive hypertrophy in a titintruncated mouse model. Biomed Res Int 2015:163564. https:// doi.org/10.1155/2015/163564

Zhou Q, Schotterl S, Backes D et al (2017) Inhibition of miR-208b improves cardiac function in titin-based dilated cardiomyopathy. Int J Cardiol 230:634-641. https://doi.org/10.1016/j.ijcar d.2016.12.171

Zhou J, Ng B, Ko NSJ et al (2019) Titin truncations lead to impaired cardiomyocyte autophagy and mitochondrial function in vivo. Hum Mol Genet. https://doi.org/10.1093/hmg/ddz033

Zou J, Tran D, Baalbaki M et al (2015) An internal promoter underlies the difference in disease severity between $\mathrm{N}$ - and C-terminal truncation mutations of Titin in zebrafish. Elife 4:e09406. https ://doi.org/10.7554/eLife.09406

Publisher's Note Springer Nature remains neutral with regard to jurisdictional claims in published maps and institutional affiliations. 